\title{
FLOW INHOMOGENEITIES IN A REALISTIC AERONAUTICAL GAS-TURBINE COMBUSTOR: FORMATION, EVOLUTION AND INDIRECT NOISE
}

\author{
Andrea Giusti, Luca Magri* \\ Department of Engineering \\ University of Cambridge \\ Trumpington Street, Cambridge \\ CB2 1PZ, United Kingdom \\ Email: Im547@cam.ac.uk
}

\author{
Marco Zedda \\ Combustion Aerothermal Methods \\ Rolls-Royce plc. \\ PO Box 31, DE24 8BJ \\ Derby, United Kingdom
}

\begin{abstract}
Indirect noise generated by the acceleration of combustion inhomogeneities is an important aspect in the design of aeroengines because of its impact on the overall noise emitted by an aircraft and the possible contribution to combustion instabilities. In this study, a realistic rich-quench-lean combustor is numerically investigated, with the objective of quantitatively analyzing the formation and evolution of flow inhomogeneities and determine the level of indirect combustion noise in the nozzle guide vane $(N G V)$. Both entropy and compositional noise are calculated in this work. A high-fidelity numerical simulation of the combustion chamber, based on the Large-Eddy Simulation (LES) approach with the Conditional Moment Closure (CMC) combustion model, is performed. The contributions of the different air streams to the formation of flow inhomogeneities are pinned down and separated with seven dedicated passive scalars. LES-CMC results are then used to determine the acoustic sources to feed an NGV aeroacoustic model, which outputs the noise generated by entropy and compositional inhomogeneities. Results show that non-negligible fluctuations of temperature and composition reach the combustor's exit. Combustion inhomogeneities originate both from finite-rate chemistry effects and incomplete mixing. In particular, the role of mixing with dilution and liner air flows on the level of combustion inhomogeneities at the combustor's exit is highlighted. The species that most contribute to indirect noise are identified and the transfer functions of a realistic NGV are computed. The noise level indicates that indirect noise generated by temperature fluctuations is larger that the indirect noise generated by compositional inhomogeneities, although the latter is not negligible and is expected to become louder in supersonic nozzles. It is also shown that relatively small fluctuations of the local flame structure can lead to significant variations of the nozzle transfer function, whose gain increases with the Mach number. This highlights the necessity of an on-line solution of the local flame structure, which is performed in this paper by $C M C$, for an accurate prediction of the level of compositional noise.

This study opens new possibilities for the identification, separation and calculation of the sources of indirect combustion noise in realistic aeronautical gas turbines.
\end{abstract}

*Address all correspondence to this author. 


\section{NOMENCLATURE}

\section{Abbreviations:}

CFL Courant-Friedrichs-Lewy

CMC Conditional Moment Closure

FDF Filtered (probability) Density Function

LES Large-Eddy Simulation

LIBSC SpeedChem Library

NGV Nozzle Guide Vane

RIDN Rear Inner Discharge Nozzle

RODN Rear Outer Discharge Nozzle

RTDF Radial Temperature Distribution Factor

SMD Sauter Mean Diameter

URANS Unsteady Reynolds-Averaged Navier-Stokes

Greek:

$\boldsymbol{\sim}$

Heat-capacity factor

$\Delta h_{f}^{0} \quad$ Formation enthalpy

$\eta \quad$ Mixture-fraction sample space for CMC

$\gamma \quad$ Heat-capacity ratio

$\mu_{i} \quad$ Chemical potential of $i$-th species

$\omega_{\alpha} \quad$ Reaction rate of the $\alpha$-th species

$\Pi \quad$ Evaporation rate source

$\pi^{ \pm} \quad$ Forward/backward acoustic waves

$\Psi \quad$ Chemical potential function

$\rho \quad$ Density

$\sigma \quad$ Entropy advected perturbation

$\xi \quad$ Mixture fraction advected perturbation, $\xi=Z^{\prime}$

Other:

$\angle(\bullet)$

se of transfer function

(•) Mean-flow quantity

(•) Fourier transform

(•) Filtered quantity

Roman:

$\bar{D} / D t^{*} \quad$ Linearized non-dimensional material derivative

$\mathcal{I} \quad$ Vector of nozzle invariants

$\boldsymbol{E} \quad$ A matrix in the aeroacoustic model

$\boldsymbol{r} \quad$ Vector of nozzle Riemann invariants

$c \quad$ Speed of sound

$c_{p} / c_{v} \quad$ Heat capacity at constant pressure/volume

$f \quad$ Frequency of the perturbation entering the nozzle

$g_{i} \quad$ Specific Gibbs' energy of $i$-th species

$h \quad$ Enthalpy

He NGV Helmholtz number

$L \quad$ NGV chord

$M \quad$ Mach number

$N \quad$ Scalar dissipation rate

$N_{s} \quad$ Number of species

p Pressure

$Q_{\alpha} \quad$ Conditionally filtered mass fraction of the $\alpha$-th species

$R \quad$ Specific gas constant

$R_{u} \quad$ Universal gas constant 


$\begin{array}{ll}s & \text { Entropy } \\ t & \text { Time } \\ t^{*} & \text { Non-dimensional aeroacoustic time } \\ T_{30} & \text { Temperature of the flow at the combustor's inlet } \\ u & \text { Axial velocity } \\ W_{i} & \text { Molar mass of } i \text {-th species } \\ x & \text { Axial coordinate } \\ x^{*} & \text { Non-dimensional nozzle axial coordinate } \\ Y_{i} & \text { Mass fraction of } i \text {-th species } \\ Z & \text { Mixture fraction } \\ Z_{i}^{(a)} & \text { Passive scalar of the } i \text {-th air stream } \\ \text { Subscripts: } \\ b & \text { NGV outlet } \\ r m s & \text { Root Mean Square } \\ s t & \text { Stoichiometric } \\ \text { Superscripts: } \\ , & \text { Linear perturbation/Fluctuation over the mean value } \\ \circ & \text { Thermochemistry reference condition }\end{array}$

\section{INTRODUCTION}

Combustion technologies used in aeronautical gas turbine combustors, based either on rich burn or lean burn concepts, usually rely on the fine control of liquid fuel preparation and mixing to achieve the targets in terms of emissions and temperature profile at the combustor's exit. The flow in a gas-turbine combustor is usually characterized by a high level of unsteadiness to ensure rapid mixing in a limited space. Although rapid mixing and formation of a homogeneous mixture allows for a better control of the temperature and emissions, the highly unsteady nature of the reacting flow, the cooling flows and the requirement of compactness could generate combustion inhomogeneities that reach the combustor's exit [1,2]. Typical examples of flow inhomogeneities are temperature inhomogeneities (also known as entropy spots) [3] and fluctuations of the mixture composition [4,5] (also known as compositional blobs). The acceleration of such inhomogeneities in the nozzle at the combustor's exit leads to the generation of pressure waves [3,5], which give a significant contribution to combustion noise. The generation of pressure waves is caused by the different acceleration that the inhomogeneity experiences with respect to the surrounding mean flow. Furthermore, the pressure waves that are reflected off the nozzle and propagate back to the flame can cause combustion instabilities (also known as thermoacoustic instabilities) [6]. Loud combustion noise and instabilities are unwanted in aero-engines. Hence, it is paramount for industrial combustors' design to investigate how combustion inhomogeneities form/evolve and quantify their acoustic contribution.

Flow inhomogeneities are generated by the unsteady nature of the combustion process and turbulent mixing and then convected towards the combustor's exit. In realistic combustors, fuel atomization, mixing and stabilization of the flame are typically achieved by means of a number of swirling flows, which interact with each other leading to the formation of recirculation regions with high turbulent fluctuations. In addition, dilution and cooling flows may locally increase the level of turbulence as well as generate inhomogeneities due to incomplete mixing. The location where such inhomogeneities are formed depends on the configuration. In some cases, the interaction with dilution and cooling flows is a key factor [1]. Non-uniform velocity profiles and turbulent mixing may decrease the strength of flow inhomogeneities as they are transported towards the combustor's exit. The dispersion and diffusion of entropy spots have been analyzed in simple geometries [7,8] and configurations close to realistic combustors [2,9] using both experiments and numerical simulations. As discussed in $[9,10]$, the role of turbulent mixing on the attenuation of entropy spots is expected to be significant in real geometries and strongly dependent on the configuration. Therefore, in order to mitigate the phenomena related to the acceleration of inhomogeneities in realistic geometries, it is important to identify the contribution of each air stream to the formation and evolution of inhomogeneities. Furthermore, to the best of the authors' knowledge, studies in indirect combustion noise on the transport of compositional inhomogeneities have not been carried out yet.

The noise originated from the acceleration of flow inhomogeneities through the nozzle guide vane, or turbine blades, downstream of the combustion chamber, is usually referred to as 'indirect noise'. This is to distinguish it from the direct component generated by the unsteady heat released by the flame. The three known sources of indirect noise are (i) temperature perturbations (also called 'entropy spots') [11-14]; (ii) vorticity perturbations [15]; and (iii) perturbations in the composition of the mixture [4,5,16]. Studies on indirect noise [3] generally agree that indirect mechanisms, in particular temperature perturbations, need to be taken into account for 
the prediction of indirect combustion noise and thermoacoustic instability. By evaluating the nozzle-transfer functions with algebraic expressions [5] and numerically integrate the differential equations (as a Boundary Value Problem) [17] of a kerosene mixture, it was shown that compositional noise can be an important contributor to indirect noise in lean mixtures and supersonic regimes. Fuel effects were studied by [18]. The general theory, which considers fluctuations in the heat capacities as well, is presented in [16], where a mathematical solution of the multicomponent aeroacoustic problem was proposed. In order to characterize the compositional inhomogeneities for the aeroacoustic calculations, $[5,17,18]$ modeled the complex combustion dynamics and mixing with simple flamelet calculations.

Compositional noise was experimentally shown by [19], where air-helium compositional blobs accelerated through choked orifices were studied. Time resolved pressure measurements were compared with the pressure predicted by a 1D acoustic model. It was found that a good agreement between measurements and numerical predictions was possible only by including compositional noise in the acoustic model through the transfer functions of [5], thereby showing the importance of compositional noise and, at the same time, providing a validation to the models developed in [5]. Although all these studies indicated the relevance of compositional noise as an indirect combustion noise mechanism, there is still a need to evaluate its effects in realistic combustor geometries and at engine relevant conditions. Furthermore, the relative importance compared to indirect entropy noise has to be accurately assessed.

The objectives of this work are to quantitatively and accurately: (i) analyze the formation of flow inhomogeneities in a realistic aeroengine configuration with high-fidelity simulations (Large-Eddy Simulation with Conditional Moment Closure combustion model); (ii) calculate the evolution of such inhomogeneities and the contribution of the different air streams to the level of fluctuations at the combustor's exit; and (iii) estimate and identify the inhomogeneities and species that contribute the most to the level of indirect combustion noise in a turbine nozzle. Since combustion inhomogeneities, such as entropy spots, may also determine low frequency thermoacoustic instabilities (rumble) [1], the analysis performed here may also give useful indications and suggestions to mitigate the occurrence of such instability phenomena. Indirect noise generated by vorticity perturbations is not studied because it is usually negligible in these configurations [3].

The paper is organized as follows. First, the realistic combustor is presented. Second, the numerical methods used for the analysis of flow inhomogeneities inside the combustor and the computation of the noise level at the exit of the turbine nozzle are introduced. Third, a discussion on the results is proposed. Conclusions and recommendations for future work close the paper.

\section{COMBUSTOR CONFIGURATION}

In order to provide a reliable estimation of the level of inhomogeneities that can be found in practical applications, a realistic RollsRoyce aero-engine combustor is investigated in this work. This configuration is based on the Rich-Quench-Lean (RQL) concept. The low level of emissions is achieved through a rich flame in the primary region of the combustor followed by an intense mixing with the air coming from the dilution ports, which leads to a lean mixture in the downstream part of the combustor. Figure 1 shows a schematic of the geometry, where the relevant air streams entering the combustion chamber are highlighted. The injector, based on an airblast configuration, is characterized by three swirling streams. The atomizing edge is located in between the innermost (1) and middle (2) streams. Such flows are designed to ensure an efficient atomization of the liquid fuel and provide flame stabilization in the primary region through the formation of a recirculation zone. Dilution ports (streams (4) and (5)) are located in the so-called 'quenching region', immediately downstream of the primary reacting zone. Further air streams enter the combustion chamber through the cooling systems for the dome (6) and the outer and inner annuli (7), as well as the rear inner and outer discharge nozzles (7), immediately upstream of the nozzle guide vane $(\mathrm{NGV})$. Fuel preparation/location, combustion and mixing with all the air streams and reacting mixture are crucial aspects for the design and operability of the combustor. Their contribution to the development of combustion inhomogeneities will be investigated in this work. A high-power operating condition is considered in this investigation.

\section{METHODS}

The computational approach used in this work is schematically shown in Fig. 2. First, the formation and evolution of combustion inhomogeneities inside the combustion chamber is studied by Large-Eddy Simulation (LES) with the Conditional Moment Closure (CMC) combustion model [20]. Second, inhomogeneities and mean quantities at the combustor's exit become the inputs of a quasi-1D model of the NGV for noise prediction. This model, based on a multicomponent solver (invariant formulation), enables the assessment of the effect and level of combustion inhomogeneities on the indirect combustion noise at the exit of the NGV [16,17]. In detail, the solver takes as inputs the mean-flow Mach number, the flow parameters and the Helmholtz number, which is the non-dimensional frequency of the incoming perturbation. The outputs are the Riemann-decomposed pressure waves, entropy and compositional inhomogeneities. The ratio between an output and a non-trivial input is the nozzle transfer function. The noise level predicted by this aeroacoustic model, in the limit of compact nozzle [5], compared favorably with the indirect noise experimentally measured by [19] with air-helium compositional 
blobs accelerated through choked orifices. In the following sections, the LES-CMC approach and the aeroacoustic model for the nozzle are introduced.

\section{LES-CMC modeling of the combustor}

Turbulent mixing and its interaction with the flame and evaporation processes are crucial aspects for the prediction of fuel distribution and the formation and evolution of inhomogeneities inside the combustion chamber. The local composition and temperature is affected by turbulent fluctuations of the flow field leading to time variations of the local air-to-fuel ratio. Furthermore, turbulent transport as well as micro-mixing and evaporation process also affect the local flame structure [21,22], through the interaction with the chemical reactions at the flame-scale level. These effects are generally important in regions characterized by high scalar dissipation rate (e.g., near the fuel injection location) and close to the ports for cooling flows and injection of fresh air, where inert mixture is mixed with the reacting flow [23]. In order to properly predict all these interactions, the LES-CMC approach is used in this work. A reliable solution of the mixing field is achieved by LES, while the CMC model $[20,24]$ allows for the solution of the time evolution of the local flame structure with all the relevant physical phenomena affecting the local turbulent combustion included in the formulation. The current formulation of the LES-CMC approach was assessed and validated through simulations of lab-scale flames (e.g. [21, 25]) and gas-turbine model combustor at engine-relevant conditions [23]. These studies showed good accuracy in capturing the mean flame shape and finite-rate chemistry effects leading to local extinction and blow-off in both gaseous and spray flames [26,27].

CMC model. The CMC model is based on the solution of reacting scalars conditioned on the mixture fraction. The CMC equation for the conditionally filtered mass fraction of a generic species $Q_{\alpha}=\widetilde{Y_{\alpha} \mid \eta}$ can be written as [28]

$$
\begin{aligned}
& \frac{\partial Q_{\alpha}}{\partial t}+\underbrace{\widetilde{u_{i} \mid \eta} \frac{\partial Q_{\alpha}}{\partial x_{i}}}_{T 1}=\underbrace{e_{\alpha}}_{T 2}+\underbrace{\widetilde{N \mid \eta} \frac{\partial^{2} Q_{\alpha}}{\partial \eta^{2}}}_{T 3}+\underbrace{\widetilde{\omega_{\alpha} \mid \eta}}_{T 4} \\
& +\underbrace{\delta_{\alpha, f} \widetilde{\Pi \mid \eta}-\left(Q_{\alpha}+(1-\eta) \frac{\partial Q_{\alpha}}{\partial \eta}\right) \widetilde{\Pi \mid \eta}}_{T 5}
\end{aligned}
$$

where $\eta$ is the mixture fraction sample space variable, and $\widetilde{u_{i} \mid \eta}, \widetilde{N \mid \eta}, \widetilde{\omega_{\alpha} \mid \eta}$ and $\widetilde{\Pi \mid \eta}$ are the conditionally filtered velocity, scalar dissipation rate, reaction rate and evaporation source, respectively. $\delta_{\alpha, f}$ is equal to 1 for the fuel and 0 for all the other species, $t$ represents the time and $x_{i}$ is the $i$-th space coordinate.

The terms $T 1$ and $T 2$ in Eqn. (1) represent the transport in the physical space. The sub-grid scale contribution, $e_{\alpha}$, is closed by a gradient assumption [25], neglecting the sub-grid joint fluctuation of species mass fraction and droplet evaporation rate [28, 29], whereas the conditionally filtered velocity is modeled as $\widetilde{u_{i} \mid \eta}=\widetilde{u_{i}}$. The term $T 3$ represents the micro-mixing with $\widetilde{N \mid \eta}$ modeled using the Amplitude Mapping Closure model [30] considering contributions from both the resolved and sub-grid scales [21]. $\omega_{\alpha} \mid \eta$ (term $T 4$ in Eqn. (1)) is closed by a first order approximation. In order to properly capture turbulence-chemistry interactions and their effect on the flame structure, a detailed chemical mechanism for kerosene (modeled as dodecane) with 38 species is employed [23,31]. Furthermore, the use of a detailed chemical mechanism also improves the accuracy of the prediction on indirect noise transfer functions, which, as explained later, depend on the composition and thermo-chemical properties of the reacting mixture. The effect of evaporation on the local flame structure is included in the formulation through the terms $T 5$, closed with the models discussed in [21, 28]. An equation similar to Eqn. (1), without chemical source term, is solved for the conditionally filtered enthalpy. Unconditional values of temperature, density and species mass fractions are computed from the respective conditional values through integration over the mixture fraction Filtered probability Density Function (FDF). The FDF is modeled by a presumed $\beta$-function, which is computed from the resolved mixture fraction and its sub-grid scale variance, which, in turn, are computed by transport equations with spray source terms included [21]. More details regarding the formulation that is adopted in this work can be found in [23] and references therein.

Computational setup. The LES solution of the mixing field (filtered velocity, filtered mixture fraction and sub-grid scale mixture fraction variance) is computed with the Rolls-Royce code PRECISE-UNS [32], which exploits a low-Mach Eulerian-Lagrangian formulation for dilute sprays. The CMC equations are solved with an in-house unstructured code [25]. The coupling between the LES 
and CMC solvers is achieved through density and temperature, with the CMC solver that receives the flow quantities from the LES (including spray source terms) and returns the unconditional temperature and density to the LES solver.

The computational domain consists of one sector of the annular combustor and includes both the inner and outer annuli, as schematically shown in Fig. 1. The injector is only partially included in the domain, with the inlet boundary of the various swirling flows placed at the exit of the corresponding swirling vanes. This choice was made to enable the injection of different passive scalars for each of the injector air streams, thereby pinning down their contribution to the mixing field and formation of inhomogeneities. The LES equations are solved in a hexa-dominant mesh of about 26 million cells with a minimum grid size of about $0.3 \mathrm{~mm}$ in the flame region. Pope criterion [33] was used as an indicator of grid quality for LES, and the resolution was found to be sufficient to resolve at least $85 \%$ of the turbulence kinetic energy in the entire combustion chamber. Following common practice [30], the CMC equations are solved in a coarser mesh of about 42,000 cells. Transfer of quantities from the LES to the CMC resolution is based on a FDF-weighted average [30]. A velocity profile, which was obtained from a URANS computation of the full system, is imposed at the inlet of each air stream. In order to investigate the contribution of each air flow to the formation and transport of inhomogeneities, different passive scalars (value equal to 1 at the corresponding inlet and 0 at all the other inlets) are injected at the inlets of the various flows. Seven passive scalars are transported in the LES simulation. The various air inlets are grouped as schematically shown in Fig. 1. This choice is made to distinguish the contribution of the three injector flows (streams 1 to 3), the dilution air through the outer and inner and inner annuli (streams 4 and 5 , respectively), dome cooling (6) and air streams through the outer and inner liners (7), including flows from the liner cooling slots and rear inner and outer discharge nozzles (RIDN and RODN). The local value of each passive scalar corresponds to the local mass fraction of the flow coming from the corresponding stream. No-slip adiabatic condition is used for all the other solid boundaries whereas an outlet condition is imposed at the outlet boundary, which is located downstream of the actual combustor's exit. 51 nodes are used to discretize the mixture fraction space. The nodes are clustered around the stoichiometric mixture fraction $\left(\eta_{s t} \approx 0.063\right)$. Pure air at $T_{30}$ is imposed at $\eta=0$, whereas pure vaporised fuel at saturation conditions is imposed at $\eta=1$. A zero-gradient condition is used at the outlet and solid walls for the CMC equations. The inert mixing solution is imposed at every air inlet. Second-order accurate numerical schemes, both in space and time, are utilized in the LES. An operator splitting technique $[25,28]$ is used for the solution of the CMC equations, as detailed in Ref. [23]. Integration of the chemistry is performed using LIBSC [34] with analytical Jacobian. A time step of $2 \mu \mathrm{s}$ is set for both the CMC and LES solvers. This makes the CFL number lower than unity in the whole flame region.

Droplet diameters are sampled from a Rosin-Rammler distribution with SMD and dispersion parameter based on previous studies (Rolls-Royce confidential). Droplet evaporation is modeled with the Abramzon and Sirignano model [35] with an infinite conductivity assumption for the liquid. Typical drag relations for spherical particles are adopted. No secondary breakup is included in the simulation due to the low value of the Weber number. The simulation is initialized from a preliminary URANS computation. Statistics are collected for 2 flow-through times, after 2.5 flow through times from the initialization of the LES. The simulation was performed on $1282.6 \mathrm{GHz}$ processors with 4GB of RAM per processor. The computation of $1 \mathrm{~ms}$ of physical time requires almost $18 \mathrm{~h}$.

\section{Aeroacoustic model of the nozzle guide vane (NGV)}

With the modeling strategy shown in Fig. 2, the flow mixture exiting the combustion chamber becomes the input of the aeroacoustic model of the nozzle guide vane (NGV). The multicomponent compressible flow inside the nozzle is governed by the conservation of mass, momentum, energy and species, respectively $[16,36]$

$$
\begin{aligned}
\frac{D \rho}{D t}+\rho \frac{\partial u}{\partial x} & =0, \\
\rho \frac{D u}{D t}+\frac{\partial p}{\partial x} & =0, \\
T \frac{D s}{D t} & =-\sum_{i=1}^{N_{s}} \frac{\mu_{i}}{W_{i}} \frac{D Y_{i}}{D t}, \\
\frac{D Y_{i}}{D t} & =0
\end{aligned}
$$


equipped with Gibbs' equation for multicomponent gases

$$
T d s=d h-\frac{d p}{\rho}-\sum_{i=1}^{N_{s}} \frac{\mu_{i}}{W_{i}} d Y_{i},
$$

where $\rho$ is the density; $u$ is the axial velocity; $p$ is the pressure; $s=\sum_{i=1}^{N_{s}} s_{i} Y_{i}$ is the specific entropy; $T$ is the temperature; $Y_{i}$ is the mass fraction of the $i$-th species, where $N_{s}$ is the number of species; $h=\sum_{i=1}^{N_{s}} h_{i} Y_{i}$ is the specific enthalpy; and $W_{i}$ is the molar mass. $\mu_{i}=W_{i} g_{i}$ is the chemical potential, and $g_{i}$ is the specific Gibbs' energy. First, this quasi-one-dimensional model assumes that, inside the nozzle, viscosity and diffusivity are negligible and the mixture is not chemically reacting. Second, the gas is assumed ideal such that $p=\rho R T$, where $R=R_{u} \sum_{i=1}^{N_{s}} Y_{i} / W_{i}$ is the mixture specific gas constant and $R_{u}$ is the universal gas constant.

Third, the gas is assumed calorically perfect such that $h=c_{p}\left(T-T^{\circ}\right)$, where $c_{p}=\sum_{i=1}^{N_{s}} c_{p, i} Y_{i}$ is the mixture specific heat capacity at constant pressure. $c_{p, i}$ is constant. The gas composition is parameterized in the mixture fraction space, $Y_{i}=Y_{i}(Z)$ [37]. On the aforementioned assumptions, Gibbs' equation becomes

$$
\frac{d s}{c_{p}}=\frac{d p}{\gamma p}-\frac{d \rho}{\rho}-(\aleph+\Psi) d Z
$$

where $\gamma=c_{p} / c_{v}$ is the heat-capacity ratio, and $c_{v}$ is the mixture specific heat capacity at constant volume. The non-dimensional terms

$$
\begin{gathered}
\Psi=\frac{1}{c_{p} T} \sum_{i=1}^{N_{s}}\left(\frac{\mu_{i}}{W_{i}}-\Delta h_{f, i}^{\circ}\right) \frac{d Y_{i}}{d Z}, \\
\boldsymbol{\aleph}=\sum_{i=1}^{N_{s}}\left(\frac{1}{(\gamma-1)} \frac{d \log (\gamma)}{d Y_{i}}+\frac{T^{\circ}}{T} \frac{d \log \left(c_{p}\right)}{d Y_{i}}\right) \frac{d Y_{i}}{d Z},
\end{gathered}
$$

are named chemical potential function and heat-capacity factor, respectively $[5,16]$. If the sensible quantities were used in (6), the term $\Delta h_{f, i}^{\circ}$ in (8) would not appear.

The equations are linearized on top of a mean flow, which is denoted by an overbar $\overline{(\bullet)}$. By gathering the mean-flow terms, it is found that the mean-flow has constant and uniform entropy and mixture fraction [16].

The variables are non-dimensionalized as $x^{*}=x / L$, where $L$ is the nozzle chord; $t^{*}=t f$ where $f$ is the frequency of the inhomogeneities; $\bar{M}=\bar{u} / \bar{c}$ is the mean-flow Mach number; and $\tilde{u}=\bar{u} / \bar{c}_{\text {ref }}$, where $\bar{c}_{\text {ref }}$ is a reference speed of sound.

The linearized multicomponent gas equations read [16]

$$
\begin{aligned}
& \frac{\bar{D}}{D t^{*}}\left(\frac{p^{\prime}}{\bar{\gamma} \bar{p}}\right)+\tilde{u} \frac{\partial}{\partial x^{*}}\left(\frac{u^{\prime}}{\bar{u}}\right)-\frac{\bar{D} \bar{\Phi}}{D t^{*}} Z^{\prime}=0, \\
& \frac{\bar{D}}{D t^{*}}\left(\frac{u^{\prime}}{\bar{u}}\right)+\frac{\tilde{u}}{\bar{M}^{2}} \frac{\partial}{\partial x^{*}}\left(\frac{p^{\prime}}{\bar{\gamma} \bar{p}}\right)+\left[2 \frac{u^{\prime}}{\bar{u}}+(1-\bar{\gamma}) \frac{p^{\prime}}{\bar{\gamma} \bar{p}}\right. \\
& \left.\quad-\frac{s^{\prime}}{\bar{c}_{p}}-(\bar{\Psi}+\overline{\boldsymbol{\kappa}}+\bar{\Phi}) Z^{\prime}\right] \frac{d \tilde{u}}{d x^{*}}=0, \\
& \frac{\bar{D}}{D t^{*}}\left(\frac{s^{\prime}}{\bar{c}_{p}}\right)=0, \\
& \frac{\bar{D} Z^{\prime}}{D t^{*}}=0
\end{aligned}
$$

where ' denotes a small fluctuation, and $\bar{\Phi}=\frac{d \log (\bar{\gamma})}{d Z} \log \left(\bar{p}^{\frac{1}{\bar{\gamma}}}\right)$ is the $\gamma$-source of noise [38], which is negligible here because of the logarithmic dependence [16]. The linearized material derivative is defined as $\bar{D}(\cdot) / D t^{*}=H e \partial(\cdot) / \partial t^{*}+\tilde{u} \partial(\cdot) / \partial x^{*}$, where $H e=f L / \bar{c}_{r e f}$ 
is the Helmholtz number, which is the ratio between the inhomogeneity and acoustic wavelengths. Equation (10) shows the sources of indirect noise. The unsteady interaction between the specific entropy perturbation, $s^{\prime}$, the compositional perturbation $Z^{\prime}$ and the mean-flow gradient, $d \tilde{u} / d x^{*}$, is the source of indirect noise.

The Riemann invariants of the aeroacoustic system are

$$
\pi^{ \pm}=\frac{1}{2}\left(\frac{p^{\prime}}{\bar{\gamma} \bar{p}} \pm \frac{u^{\prime}}{\bar{c}}\right) \quad \sigma=\frac{s^{\prime}}{\bar{c}_{p}} \quad \xi=Z^{\prime}
$$

The superscripts $+/-$ denote waves that travel in the same/opposite direction of the mean flow. The variables are expressed as functions of the invariants of the compact-nozzle solution $(H e=0)$. When $H e=0$, the normalized mass flow rate, $I_{\dot{m}}$, total specific enthalpy, $I_{h_{T}}$, specific entropy, $I_{s}$, and mixture fraction, $I_{Z}$, are conserved. (The total specific enthalpy is $h_{T}=h+1 / 2 u^{2}$.) The flow invariants $\mathcal{I}=\left[I_{\dot{m}}, I_{h_{T}}, I_{s}, I_{z}\right]^{T}$ are provided by a linear transformation of the Riemann invariants, $\boldsymbol{r}=\left(\pi^{+}, \pi^{-}, \sigma, \xi\right)$ as $\mathcal{I}=\boldsymbol{D} \boldsymbol{r}$, where the matrix $\boldsymbol{D}$ is provided by Eqn. (3.1) in [16]. When Fourier-transformed, the equations are cast in a compact matrix form

$$
2 \pi \mathrm{i} H e \hat{I}=\boldsymbol{E}\left(x^{*}\right) \frac{d \hat{I}}{d x^{*}}+\boldsymbol{F}\left(x^{*}\right) \hat{I}_{Z}
$$

where

$$
\boldsymbol{E}\left(x^{*}\right)=-\tilde{u}\left[\begin{array}{cccc}
1 & \frac{\beta}{\bar{\gamma}-1) \bar{M}^{2}} & \frac{-1}{(\bar{\gamma}-1) \bar{M}^{2}} & \frac{-\bar{\Psi}}{(\bar{\gamma}-1) \bar{M}^{2}} \\
\frac{\bar{\gamma}-1}{\beta} & 1 & \frac{\bar{\gamma}-1}{\beta} & \frac{\bar{\gamma}-1}{\beta}(\bar{\Psi}+\overline{\boldsymbol{\kappa}}) \\
0 & 0 & 1 & 0 \\
0 & 0 & 0 & 1
\end{array}\right],
$$

where $\beta=1+0.5(\bar{\gamma}-1) \bar{M}^{2}$ for brevity. The $4 \times 4$ system in Eqn. (14) represents the quasi-1D multicomponent aeroacoustic model. The term $\boldsymbol{F}\left(x^{*}\right) \hat{I}_{Z}$ is neglected because its integral effect averages to a small number [16]. The boundary conditions are prescribed using the method of [14], which was extended to include the additional invariant, $\hat{I}_{Z}$, in [5]. Physically, the perturbations prescribed at the nozzle's inlet are generated in the combustion chamber due to the unsteady and inhomogeneous combustion process. Note that in previous studies in compositional noise [5,16-18] these inhomogeneities were estimated with simple 1D flamelet calculations. In this paper, the inhomogeneities are characterized with LES-CMC high-fidelity simulation of the realistic gas-turbine combustion chamber. The system (14) can be solved as a boundary value problem. However, the method proposed by [16] is deployed here. In this method the acoustic propagator is directly obtained by a Dyson expansion used in Quantum-Mechanics problems. The solution is given by

$$
\hat{\mathcal{I}}\left(x^{*}\right)=\boldsymbol{U}\left(x_{a}^{*}, x^{*}\right) \hat{\mathcal{I}}_{a} \text {. }
$$

With the explicit calculation of the acoustic propagator, $\boldsymbol{U}$ [16], the solution is known at any location by a simple matrix-vector multiplication.

\section{RESULTS}

The ultimate objective of this work is to assess the impact of inhomogeneities generated by the unsteady mixing and combustion on the indirect noise emitted by the nozzle. Thus, the following discussions focus on temperature inhomogeneities and compositional inhomogeneities. The analysis of formation and evolution of inhomogeneities is performed by investigating (i) the LES-CMC solution in stream-wise and axial cross sections of the combustor and (ii) the time history of relevant quantities at selected locations (probes, Fig. 4(a)) inside the combustion chamber. Finally, the quantities sampled at the combustor's exit in the LES-CMC simulation are used to feed the aeroacoustic model of the NGV to evaluate the impact of the inhomogeneities on the noise level.

Before discussing the phenomena related to the transport of inhomogeneities, the accuracy of the LES-CMC simulation is assessed 
by comparing the radial profile of the mean temperature (expressed in non-dimensional form as Radial Temperature Distribution Factor (RTDF)) with experimental measurements. Figure 3 shows the good agreement between measurements and numerical simulations.

\section{Formation and Evolution of Inhomogeneities}

Two different contributions to the formation of temperature and compositional inhomogeneities can be distinguished: (i) local fluctuation of the mixture fraction, which is affected by fuel evaporation and mixing with the turbulent air streams, and (ii) local fluctuation of the flame structure due to the effect of the spatial gradients of mixture fraction, turbulent transport and evaporation itself (see Eqn. (1)). The mean mixture fraction field and the rms of the mixture fraction and temperature fluctuations are shown in Fig. 4 (the sub-grid scale fluctuations, which are not shown here, give a negligible contribution to the rms field). In the limit of low-Mach number flows, as those inside the combustion chamber, the effect of pressure fluctuations on entropy is negligible. Therefore the remarks on the temperature fluctuations also apply to the entropy field at a constant composition. Consistently with the RQL design, the primary region of the combustor is generally characterized by rich mixture with a lean region downstream of the dilution ports. The primary combustion region is also characterized by high fluctuations of mixture fraction, as highlighted by the rms value. These strong fluctuations are mainly due to the evaporation and the intense mixing with the air flows, as also shown in Fig. 5 where the rms of the fluctuations of the various passive scalars is reported. Downstream of the dilution ports, the level of fluctuations becomes less intense. However, some residual fluctuations persist at the combustor's exit, which are the causes of indirect combustion noise. As far as the fluctuations of temperature are concerned (Fig. 4(c)), strong levels of fluctuations exist in the primary and quenching regions of the combustor, as well as along the shear layer of the different air flows. The level of fluctuation decreases moving towards the exit of the combustor even if, close to the liner walls, mixing with the cooling flows leads to a local increase of the fluctuations. Note that the local fluctuations of temperature (and species mass fractions) are the results of the local variation of both mixture fraction (and its sub-grid scale variance) and flame structure.

Figure 6 shows the flame structure in mixture fraction space computed by CMC at two selected locations. Although phenomena of local extinction are generally not observed at this condition, fluctuations of the scalar dissipation rate, which are relatively small as compared to the extinction value, and turbulent transport (including mixing with inert flow coming from the cooling and inlet ports) cause fluctuations of the flame structure. Temperature fluctuations at the stoichiometric mixture fraction can reach 5-10\% of the peak value. Fluctuations of species mass fraction (e.g. CO in Fig. 6) are observed as well. Their relative intensity depends on the specific species and it is related to the strong coupling between turbulent mixing and chemistry. This is a further contribution to the formation of temperature and species inhomogeneities. As highlighted in Fig. 6, fluctuations of the flame structure characterize the solution both in the primary region and at the combustor's exit, with higher levels of fluctuations close to the cooling ports and lower values along the centerline. The flame structure computed by the CMC is directly used for the computation of the NGV transfer functions thereby enabling the estimation of the mean strengths of the acoustic sources $\bar{\Psi}$ and $\overline{\boldsymbol{\kappa}}$. The effect of time variation of these quantities on the modulation of indirect combustion noise is beyond the scope of this paper and is left for future work.

Inhomogeneities generated by incomplete mixing and local fluctuations of the flame structure are advected downstream towards the combustor's exit. As discussed in previous work [8,9], the action of turbulent mixing decreases the intensity of the fluctuations as highlighted by the decrease of the rms of the mixture fraction and temperature fluctuations along the combustor centerline (see Fig. 4). However, close to the combustor liners, the mixing with fresh air coming from the cooling flows, as well as air inlet ports (such as RIDN and RODN), causes a local increase of the fluctuations. Shear dispersion [39], due to a non-uniform mean velocity profile, is another important phenomenon to be considered when values of inhomogeneities averaged over the cross section are considered (e.g. for low-order network modeling). The differential time delay due to a non-uniform mean velocity profile reduces the cumulative effect of the fluctuations. However, differently from studies in simplified geometries [7, 8], the presence of strong levels of turbulent fluctuations, recirculation regions and interaction with cooling flows enhances the effect of turbulent mixing when realistic combustor configurations are considered.

The mean and rms fields of the temperature and mixture fraction at the combustor's exit are shown in Fig. 7. A non-negligible level of fluctuations is present in the entire section with peaks that are close to the combustor liners owing to the injection of fresh air from the cooling systems, RIDN and RODN. Although the mean mixture fraction shows a lean region downstream of the dilution ports, the instantaneous mixture fraction field generally shows pockets of rich mixture that persist downstream of the quenching region (Fig. 8). Small spots of rich mixture sometimes reach the combustor's exit, which further contribute to the level of fluctuations at the exit plane. Furthermore, some heat-release rate (HRR) is also present at the combustor's exit. The aeroacoustic model should be extended to include the presence of heat-release in RQL combustors, which is left for future work.

A question to address is: Which are the air flows that most contribute to the level of combustion inhomogeneities at the combustor's exit? Figure 9 shows the correlation of the time evolution of the mixture fraction at Probe 18 (combustor's exit) with the time evolution of mixture fraction at all the other probes (see Fig. 4(a) for the probe locations). The same quantity is also shown for the temperature. A 
strong positive correlation exists with all the probes downstream of the dilution ports for both the mixture fraction and the temperature. This indicates that the inhomogeneities at the exit of the combustor are mainly affected by the mixing with the dilution ports. This is consistent with the numerical study performed by Motheau et al. [1] where the transport of entropy spots in a RQL configuration was investigated. They found that most of the entropy fluctuations are due to incomplete mixing caused by the dilution ports. The influence of the various air streams to the mixture fraction fluctuations at the combustor's exit is further investigated in Fig. 10, where the correlation between mixture fraction and the passive scalars associated with the different air streams is shown at different locations along the radius (probes at the exit plane are considered). By definition, $Z+\sum_{i} Z_{i}^{(a)}=1$, where $Z$ is the usual mixture fraction associated with the fuel, and $Z_{i}^{(a)}$ is the passive scalar related to each of the air streams. Since the addition of an air flow generally causes a decrease of the mass fraction associated with all the other flows, a direct effect on the mixing is denoted by a negative correlation, whereas a high positive correlation indicates that the mixing between the two streams is almost complete. At locations close to the liners, the mixture fraction fluctuations are mainly influenced by the air flows at the walls (stream 7). Moving towards an intermediate radius the influence of the dilution ports (streams 4 and 5) increases. The relative importance of cooling flows and air through the RIDN and RODN (both included in stream 7) strongly depends on the respective mass flow rate and geometry configuration. This study highlights the overall importance of these flows on the generation of inhomogeneities at the combustor's exit, thereby suggesting that a proper design of such systems can help reduce the level of mixture fraction fluctuations.

On the contrary, the correlation with the other air streams is always positive, which shows that the inhomogeneities at the combustor's exit are mainly caused by the flows through the dilution ports and the liner air flows downstream of the quenching region. The same analysis is also performed for probes located inside the primary region and immediately downstream of the dilution streams. Results are shown in Fig. 11 for two selected points. Although in the primary region all the streams contribute to the mixing, the level of fluctuations downstream of the quenching region is chiefly affected by the dilution streams. The presence of a recirculation region in the primary region of the combustor certainly help the mixing between fuel and air. Furthermore, although some residual level of unmixedness may still be present in the flow that leaves the primary region (e.g., Fig. 5), the high mass flow rate of the air introduced by the quenching ports makes such streams dominate the downstream behavior.

\section{Indirect noise emitted by the nozzle guide vane}

From Eqn. (10), it is noted that compositional indirect noise is generated by acoustic sources whose strength is proportional to the chemical potential function, $\bar{\Psi}$, and the heat-capacity factor, $\overline{\boldsymbol{\aleph}}$, which are defined in Eqn. (8). $\bar{\Phi}$ is neglected following the analysis of [16]. The compositional-source strengths are depicted in Fig. 12 at the combustor's exit.

The heat-capacity factor, $\bar{\kappa}$, may be of the same order as the chemical potential function, $\bar{\Psi}$. Physically, this means that variations in $\gamma$ and $c_{p}$ may produce as much indirect noise as variations in the Gibbs' energy. The fluctuations of the mixture fraction are depicted in Fig. 13(a).

Fluctuations in the temperature, which are sometimes called entropy spots, are shown in Fig. 13(b). The local fluctuations can become significant. In particular, close to the bottom wall (probe 22), large fluctuations of the temperature are observed. These are caused by incomplete mixing and the intrinsic fluctuations of the RIDN flows, based on a jet in cross flow configuration, located along the inner liner in the vicinity of the exit. Some residual oxidation may be further contributing to the level of temperature fluctuations. Although the local fluctuations are appreciable, the cross-sectional average is small because the fluctuations at high frequency in the entire section are weakly correlated with each other. Furthermore, Figs. 4 and 7 show that mean and rms values of temperature and mixture fraction are generally characterized by variations in space. This suggests that two- or three-dimensional effects may be important to accurately predict the nozzle aeroacoustics.

In order of importance, the species that contribute the most to the compositional acoustic strengths are $\mathrm{O}_{2}, \mathrm{CO}_{2}, \mathrm{H}_{2} \mathrm{O}$ and $\mathrm{N}_{2}$ (Fig. 14). Interestingly, the product $\mathrm{H}_{2} \mathrm{O}$ influence the acoustic strength through variations in the Gibbs' energy (panel (a)) but not through variations in $\gamma$ (panel (b)). Physically, all these species are the largest contributors because of the combined effect of their (i) large Gibbs' energy (minus the contribution from the formation enthalpy), and (ii) large gradients with respect to the mixture fraction.

Finally, the inhomogeneities are plugged into the aeroacoustic solver for the calculation of the transfer functions. The Mach-number profile of the Nozzle Guide Vane is provided by Rolls Royce (not shown). The Mach number at the outlet is studied in the range $0.7-1$. The NGV transfer functions of compositional and entropy inhomogeneities are shown in Fig. 15(a,b) and (c,d), respectively. As noticed in previous studies [14,16,17], as the Helmholtz number increases, the gain decreases, i.e. the nozzle filters out higher frequencies. The phase has a linear behavior, i.e., it is proportional to the NGV spatial extent/perturbation frequency. Although the variations of $\bar{\Psi}+\overline{\boldsymbol{\kappa}}$ are relatively small, the quasi-steady response of the nozzle is markedly affected, in particular, in the limit of compact nozzles (small $\mathrm{He}$ ). The NGV Mach number at the outlet, $M_{b}$, has a marked effect on the level of indirect noise. Small changes to the Mach number bring about relative large changes in the level of indirect noise. Indeed, compositional noise becomes even higher in supersonic nozzles [5,16]. 
Vertical bars in Fig. 15(a) indicate the dispersion of the magnitude of the compositional noise transfer function due to fluctuations of the local flame structure (e.g. Fig. 6). Although such fluctuations are relatively small, a non-negligible effect on the transfer function is observed, which calls for methods that are able to properly predict the time evolution of the local flame structure (as the CMC) for an accurate evaluation of the indirect noise.

At the NGV inlet (i.e., combustor's exit), the power density spectrum of the inhomogeneities, computed from the time history of the mixture fraction/temperature predicted by LES, has the dominant peak at $f \approx 120 \mathrm{~Hz}$ and the second peak at $f_{2} \approx 1470 \mathrm{~Hz}$ (result not shown). In the configuration under investigation, these frequencies correspond to $H e \sim 0.001$ and $H e \sim 0.01$, respectively. The amplitude of the second peak is about $25 \%$ smaller that the amplitude of the dominant peak, whereas higher-frequency peaks are at least one order of magnitude smaller. The relevant NGV Helmholtz numbers are typically small. At low-frequency perturbation, the acoustics are not affected by the spatial extent of the nozzle, i.e., the nozzle is almost compact.

For the calculation of the level of indirect noise emitted by the NGV, the time-averaged value of the acoustic sources at the combustor's exit is considered, $\bar{\Psi}+\overline{\boldsymbol{\aleph}}=-4.56$. The Fourier transforms, $\hat{Z}^{\prime}=\operatorname{FFT}(Z-\bar{Z})$, (from Fig. 4(a)) and $\hat{T}^{\prime}=\operatorname{FFT}((T-\bar{T}) / \bar{T})$, which generates the (historically-named) entropy noise (from Fig. 4(b)) are multiplied by the corresponding NGV transfer functions and integrated over the spectrum, as $\left|\int_{0}^{0.2}\left(\frac{\pi_{b}^{+}}{\xi_{a}}\right) \hat{Z}^{\prime} d H e\right|=0.033$ and $\left|\int_{0}^{0.2}\left(\frac{\pi_{b}^{+}}{\sigma_{a}}\right) \hat{T}^{\prime} d H e\right|=0.32$ to give an estimate of the indirect noise level due to compositional and temperature inhomogeneities, respectively. The ratio between compositional and entropy noise is in this case $\approx 10 \%$. This result is consistent with the results obtained from flamelet calculations in subsonic/sonic nozzles [5]. When the flow becomes supersonic, compositional noise has the same order of magnitude [5,17], and may even exceed the level of indirect noise from temperature fluctuations if a shock is present in the divergent. Therefore, it is emphasized that the ratio between compositional and entropy noise can significantly change in other configurations, in particular in higher-Mach number nozzles and leaner mixtures.

\section{CONCLUSIONS}

A realistic Rolls-Royce combustor based on Rich-Quench-Lean (RQL) technology is investigated to characterize the formation/evolution of combustion inhomogeneities and their contribution to indirect noise. First, Large-Eddy simulation with Conditional Moment Closure combustion model (LES-CMC) is used to calculate the reacting flow dynamics, mixing and transport of inhomogeneities. Second, the LES-CMC results at the combustor's exit become the inputs for a quasi-one-dimensional multicomponent aeroacoustic solver for the calculation of the indirect noise emitted by the Nozzle Guide Vane (NGV). The key findings of this study are:

1. At the combustor's exit there are non-negligible fluctuations of composition and temperature (Figs. 4,7 and 13);

2. Compositional inhomogeneities originate both from incomplete mixing between fuel and air streams (which is mostly a non-reacting phenomenon, Figs. 5, 8), and fluctuations of the local flame structure (Fig. 6);

3. The level of inhomogeneity at the combustor's exit is most affected by the mixing with dilution holes and liner air flows. The latter are particularly responsible for generating fluctuations close to the liners (Figs. 7-11);

4. The species that most contribute to indirect noise are identified, i.e., in order of importance $\mathrm{O}_{2}, \mathrm{CO}_{2}, \mathrm{H}_{2} \mathrm{O}$ and $\mathrm{N}_{2}$ (Fig. 14);

5. The transfer functions of a realistic subsonic/sonic Nozzle Guide Vane (NGV) multiplied by the realistic fluctuations indicate that the level of indirect noise generated by temperature fluctuations is larger that the indirect noise generated by compositional inhomogeneities, although the latter is not negligible (Fig. 15). Compositional noise is expected to become louder in supersonic nozzles;

6. Relatively small fluctuations of the local flame structure can lead to significant variations of the nozzle transfer function, which increases with the Mach number (Fig. 15). Therefore, for an accurate prediction of the level of compositional noise, a time resolved solution of the flame structure (as in the CMC approach used here) has to be preferred.

In the combustor investigated in this work, temperature and composition fluctuations are correlated through the mixture fraction. Therefore, typical strategies that are used to mitigate entropy noise might also reduce compositional noise. However, this requires further investigation. The results and methods proposed in this study open up new possibilities for the design of quieter aero-engines.

\section{ACKNOWLEDGMENT}

Support by Rolls-Royce plc. is gratefully acknowledged by the authors. Luca Magri gratefully acknowledges the support from the Royal Academy of Engineering Research Fellowships Scheme. The authors thank Prof. E. Mastorakos for fruitful discussions and comments on this work. 


\section{REFERENCES}

[1] Motheau, E., Nicoud, F., and Poinsot, T., 2014. "Mixed acoustic-entropy combustion instabilities in gas turbines". J. Fluid Mech., 749, pp. 542-576.

[2] Wassmer, D., Schuermans, B., Paschereit, C. O., and Moeck, J. P., 2017. "An acoustic time-of-flight approach for unsteady temperature measurements: Characterization of entropy waves in a model gas turbine combustor". J. Eng. Gas Turbines Power, 139.

[3] Dowling, A. P., and Mahmoudi, Y., 2015. "Combustion noise”. Proc. Combust. Inst., 35, pp. 65-100.

[4] Ihme, M., 2017. "Combustion and Engine-Core Noise". Annual Review of Fluid Mechanics, 49(1).

[5] Magri, L., O’Brien, J., and Ihme, M., 2016. "Compositional inhomogeneities as a source of indirect combustion noise". Journal of Fluid Mechanics, 799, p. R4.

[6] Poinsot, T., 2017. "Prediction and control of combustion instabilities in real engines". Proc. Combust. Inst., 36(1), pp. 1 - 28.

[7] Morgans, A. S., Goh, C. S., and Dahan, J. A., 2013. "The dissipation and shear dispersion of entropy waves in combustor thermoacoustics". J. Fluid Mech., 733, p. R2.

[8] Giusti, A., Worth, N. A., Mastorakos, E., and Dowling, A. P., 2017. "Experimental and numerical investigation into the propagation of entropy waves". AIAA J., 55(2), pp. $446-458$.

[9] Xia, Y., Duran, I., Morgans, A. S., and Han, X., 2017. "Dispersion of entropy perturbations transporting through an industrial gas turbine combustor". Flow, Turbul. Combust.

[10] Giusti, A., 2017. "Advances in the modelling of dispersion and diffusion of entropy waves in gas-turbine combustors". Proceedings of ICSV24, London (UK).

[11] Cuadra, E., 1967. "Acoustic Wave Generation by Entropy Discontinuities Flowing Past an Area Change". The Journal of the Acoustical Society of America, 42(4), p. 725.

[12] Marble, F. E., and Candel, S. M., 1977. "Acoustic disturbance from gas non-uniformities convected through a nozzle". J. Sound Vib., 55(2), pp. 225-243.

[13] Bake, F., Richter, C., Mühlbauer, C., Kings, N., Röhle, I., Thiele, F., and Noll, B., 2009. “The entropy wave generator (EWG): A reference case on entropy noise". J. Sound Vib., 326, pp. 574-598.

[14] Duran, I., and Moreau, S., 2013. "Solution of the quasi-one-dimensional linearized Euler equations using flow invariants and the Magnus expansion". J. Fluid Mech., 723, pp. 190-231.

[15] Howe, M. S., and Liu, J. T. C., 1977. "The generation of sound by vorticity waves in swirling duct flows". Journal of Fluid Mechanics, 81, pp. 369-383.

[16] Magri, L., 2017. "On indirect noise in multi-component nozzle flows". J. Fluid Mech., 828, p. R2.

[17] Magri, L., O’Brien, J. D., and Ihme, M., 2018. "Effects of nozzle Helmholtz number on indirect combustion noise by compositional perturbations". J. Eng. Gas Turbines Power, 140, pp. 031501-031501-9.

[18] O'Brien, J. D., and Ihme, M., 2017. "Species Dependency of the Compositional Indirect Noise Mechanism". Proc. ASME 2017 Turbo Expo, GT2017-63076.

[19] Rolland, E. O., De Domenico, F., and Hochgreb, S., 2017. "Direct and indirect noise generated by injected entropic and compositional inhomogeneities". Proc. ASME Turbo Expo, GT2017-64428.

[20] Klimenko, A., and Bilger, R., 1999. "Conditional moment closure for turbulent combustion”. Prog. Energy Combust. Sci., 25(6), pp. $595-687$.

[21] Giusti, A., and Mastorakos, E., 2017. "Detailed chemistry LES/CMC simulation of a swirling ethanol spray flame approaching blow-off". Proc. Combust. Inst., 36(2), pp. 2625-2632.

[22] Olguin, H., and Gutheil, E., 2014. "Influence of evaporation on spray flamelet structures". Combust. Flame, 161(4), pp. 987 996.

[23] Giusti, A., Mastorakos, E., Hassa, C., Heinze, J., Magens, E., and Zedda, M., 2018. "Investigation of Flame Structure and Soot Formation in a Single Sector Model Combustor Using Experiments and Numerical Simulations Based on the Large Eddy Simulation/Conditional Moment Closure Approach”. J. Eng. Gas Turbines Power, 140.

[24] Mortensen, M., and Bilger, R. W., 2009. "Derivation of the conditional moment closure equations for spray combustion". Combust. Flame, 156(1), pp. $62-72$.

[25] Zhang, H., Garmory, A., Cavaliere, D. E., and Mastorakos, E., 2015. "Large Eddy Simulation/Conditional Moment Closure modeling of swirl-stabilized non-premixed flames with local extinction". Proc. Combust. Inst., 35(2), pp. 1167 - 1174.

[26] Zhang, H., and Mastorakos, E., 2016. "Prediction of Global Extinction Conditions and Dynamics in Swirling Non-premixed Flames Using LES/CMC Modelling". Flow, Turbul. Combust., 96(4), pp. 863-889.

[27] Giusti, A., and Mastorakos, E., 2016. "Numerical investigation into the blow-off behaviour of swirling spray flames using the 
les/cmc approach". Proceeding of the ETMM-11, Palermo (Italy).

[28] Tyliszczak, A., Cavaliere, D. E., and Mastorakos, E., 2014. "LES/CMC of Blow-off in a Liquid Fueled Swirl Burner". Flow, Turbul. Combust., 92(1), pp. 237-267.

[29] Ukai, S., Kronenburg, A., and Stein, O., 2015. "Large eddy simulation of dilute acetone spray flames using cme coupled with tabulated chemistry". Proc. Combust. Inst., 35(2), pp. 1667 - 1674.

[30] Triantafyllidis, A., and Mastorakos, E., 2010. "Implementation Issues of the Conditional Moment Closure Model in Large Eddy Simulations". Flow, Turbul. Combust., 84(3), pp. 481-512.

[31] Nehse, M., Warnatz, J., and Chevalier, C., 1996. "Kinetic modeling of the oxidation of large aliphatic hydrocarbons". Proc. Combust. Inst., 26, pp. 773-780.

[32] Anand, M. S., Eggels, R., Staufer, M., Zedda, M., and Zhu, J., 2013. "Derivation of the conditional moment closure equations for spray combustion". Proc. ASME Gas Turbine India Conference, GTINDIA2013-3537.

[33] Pope, S. B., 2000. Turbulent Flows. Cambridge Univ. Press, Cambridge, UK.

[34] Perini, F. The speedchem project, http://www.federicoperini.info/speedchem. Accessed: 2016-12-08.

[35] Abramzon, B., and Sirignano, W., 1989. "Droplet vaporization model for spray combustion calculations". Int. J. Heat Mass Transfer, 32(9), pp. $1605-1618$.

[36] Chiu, H. H., and Summerfield, M., 1974. "Theory of combustion noise”. Acta Astronautica, 1, pp. 967-984.

[37] Williams, F. A., 1985. Combustion Theory. Perseus Books, Reading, MA.

[38] Strahle, W. C., 1976. "Noise produced by fluid inhomogeneities". AIAA Journal, 14(7), pp. 985-987.

[39] Sattelmayer, T., 2003. "Influence of the Combustor Aerodynamics on Combustion Instabilities From Equivalence Ratio Fluctuations”. J. Eng. Gas Turbines Power, 125(1), pp. 11-19. 


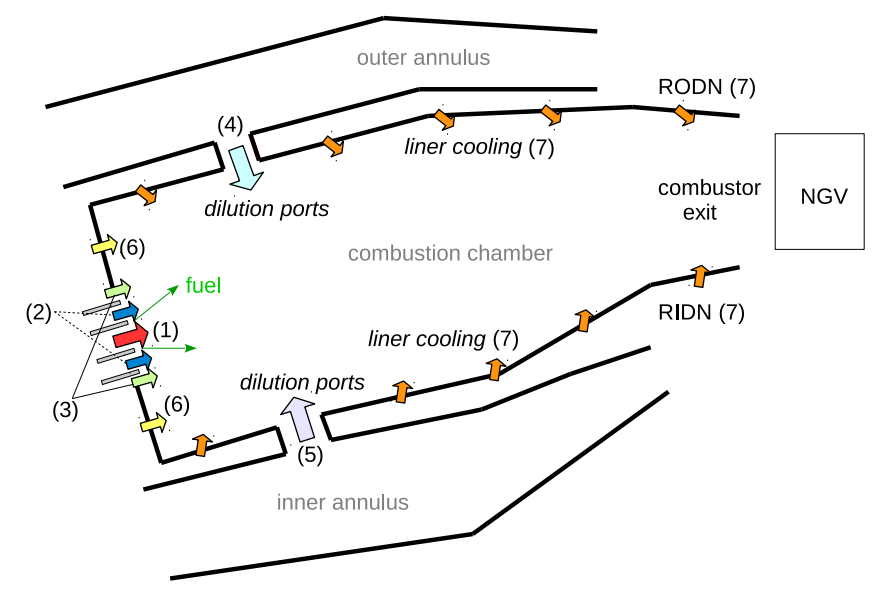

FIGURE 1: Schematic of the combustor. The arrows represent the different streams entering the combustion chamber.

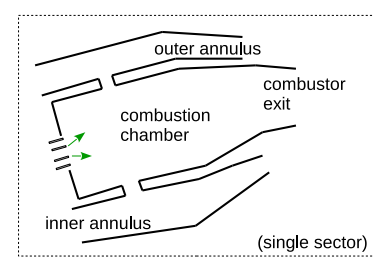

LES-CMC simulation

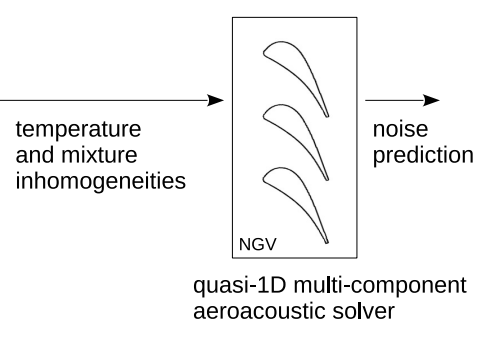

aeroacoustic solver

FIGURE 2: Schematic of the computational approach used in this work.

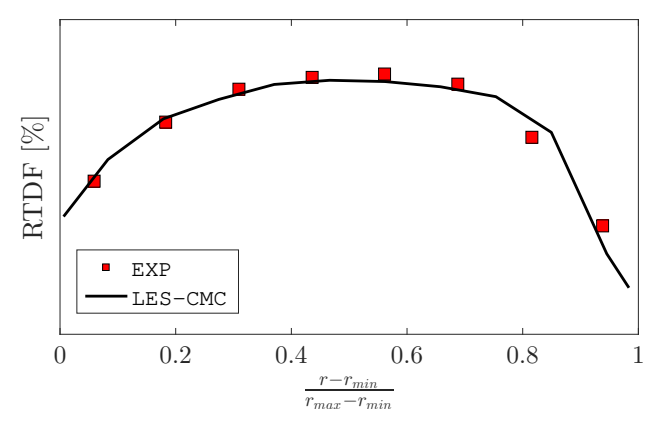

FIGURE 3: Non-dimensional radial temperature profile at the combustor's exit from experiments (EXP) and LES-CMC computation. The experimental data is provided by Rolls-Royce plc. 
(a) $\bar{Z}$

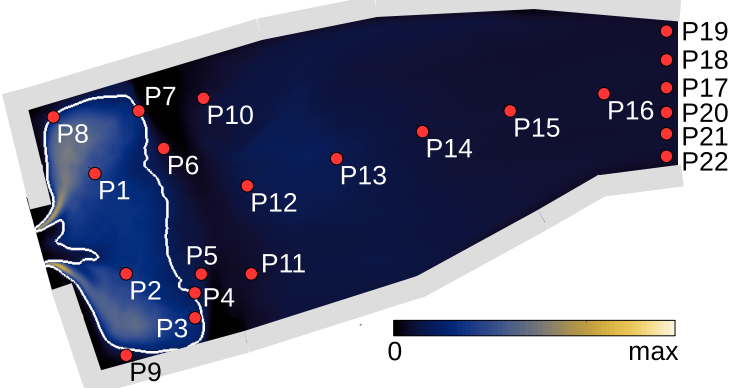

(b) $Z_{r m s}^{\prime}$

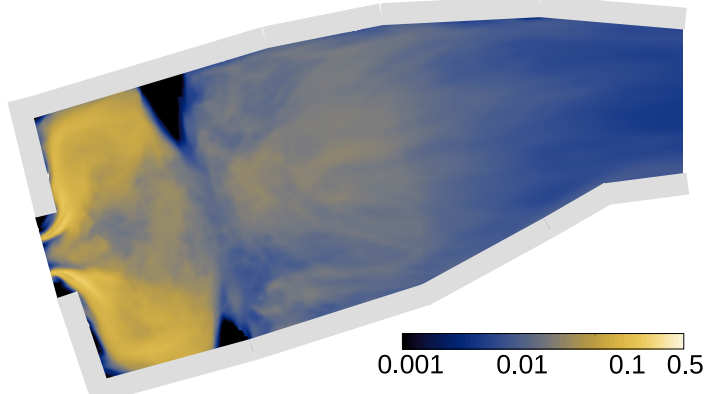

(c) $\mathrm{T}^{\prime}{ }_{r m s}(\mathrm{~K})$

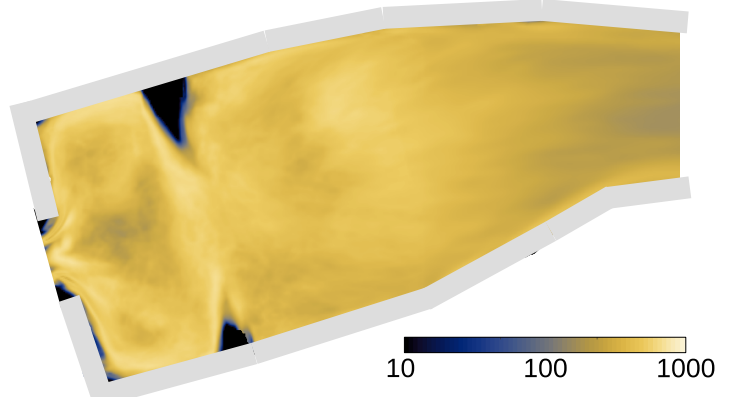

FIGURE 4: LES-CMC solution in a stream-wise cross section: (a) mean mixture fraction; (b) rms of the mixture fraction fluctuations; (c) $r m s$ of the temperature fluctuations. The white iso-line indicates the stoichiometric mixture fraction. The locations of the probes used for the analysis of local quantities is shown in (a). 
(a) $Z_{1, r m s}^{(a)}$

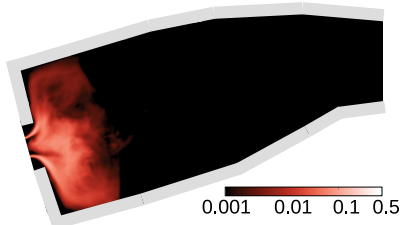

(c) $Z_{3, r m s}^{(a)}$

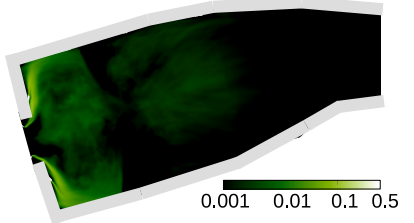

(e) $Z_{5, r m s}^{(a)}$

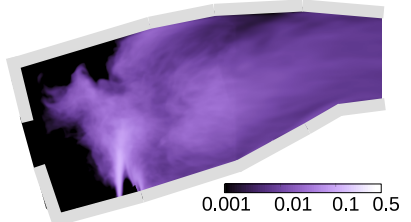

(b) $Z_{2, r m s}^{(a)}$

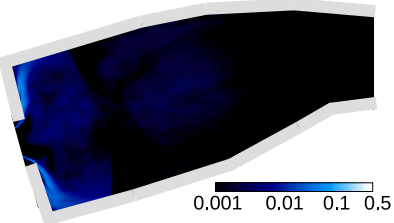

(d) $Z_{4, r m s}^{\prime(a)}$

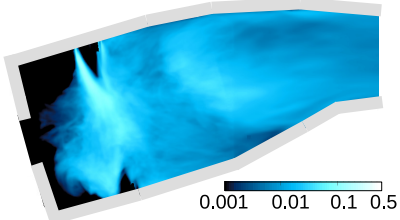

(f) $Z^{\prime(a)} 7, r m s$

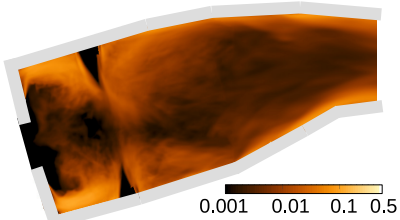

FIGURE 5: rms fields of the fluctuations of the passive scalars $Z_{i}^{(a)}$ associated with the air flows (see Fig. 1).
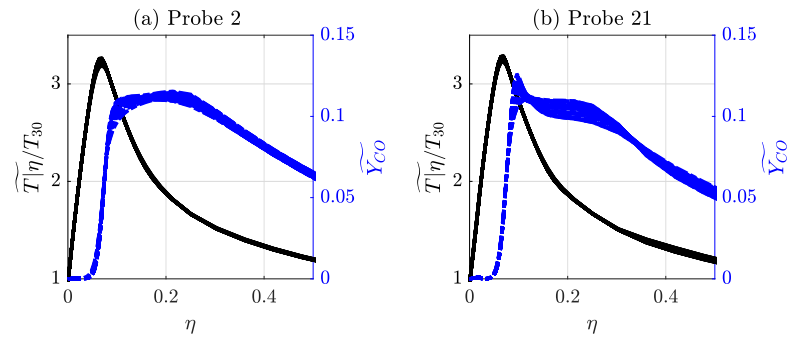

FIGURE 6: Conditionally filtered temperature (black line, left axis) and conditionally filtered CO mass fraction (blue line, right axis) in the mixture fraction space computed by LES-CMC at probe (a) 2 (primary region) and (b) 21 (combustor's exit). Each line corresponds to a different time, which is uniformly sampled over a period of $4 \mathrm{~ms}$.

(a) $\bar{Z}$

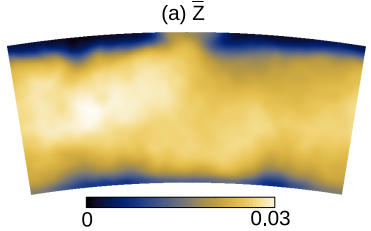

(b) $\overline{\mathrm{T}} / \mathrm{T}_{30}$

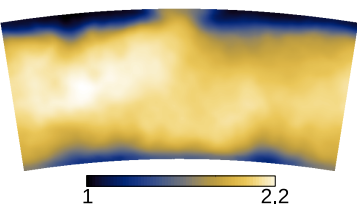

(c) $Z_{r m s}^{\prime}$

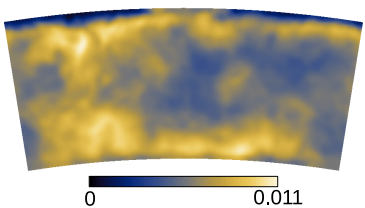

(d) $T^{\prime}{ }_{r m s} / T_{M}$

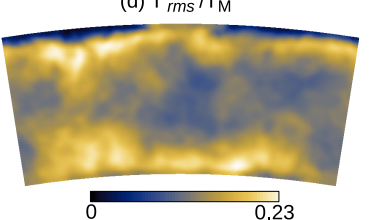

FIGURE 7: Mean and rms of the fluctuations of temperature and mixture fraction at the combustor's exit ( $T_{M}$ is the mean temperature averaged over the cross-section and $T_{30}$ is the temperature at the inlet of the combustor). 
(a) Z

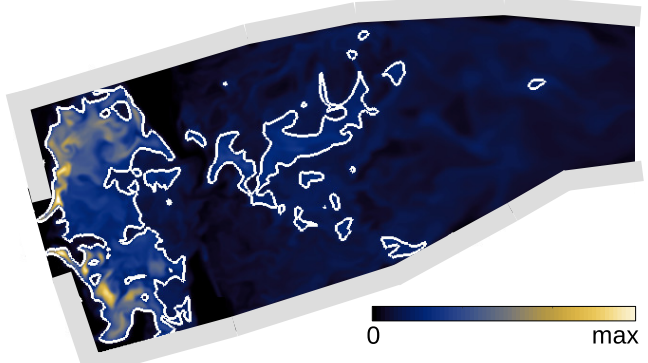

(b) HRR

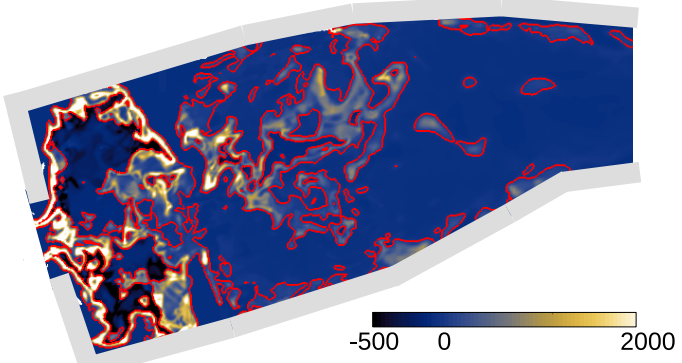

(c) HRR at the combustor's exit

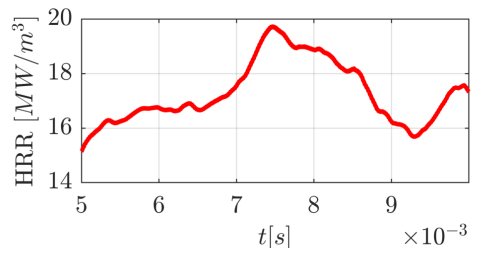

FIGURE 8: Instantaneous LES-CMC solution in a stream-wise cross section: (a) mixture fraction, and (b) heat release rate in $\mathrm{MW} / \mathrm{m}^{3}$. (c) Time history of the heat release rate integrated over the combustor's exit plane. In (a), the white line is the stoichiometric mixture fraction. In (b), the red line is the iso-line of $\mathrm{HRR}=100 \mathrm{MW} / \mathrm{m}^{3}$.

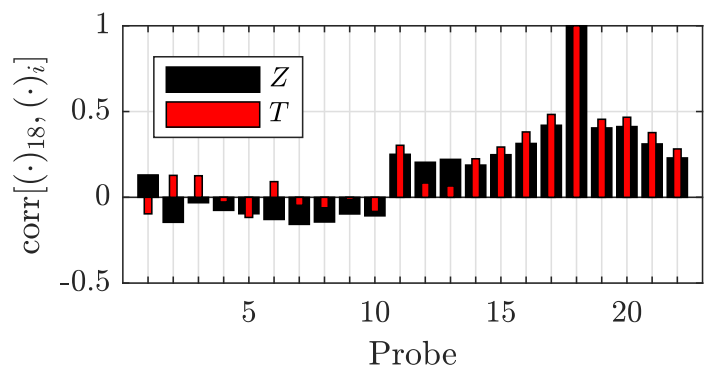

FIGURE 9: Correlation between the mixture fraction, $Z$, of probe 18 at the combustor's exit and the mixture fraction of the other probes (see Fig. 4(a) for the numbering). In red, the same quantity is shown for the temperature. 


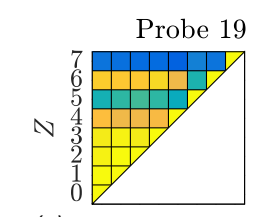

(a) 01234567

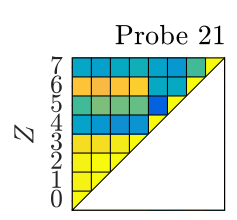

(c) 01234567

Z

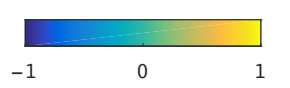

Probe 17

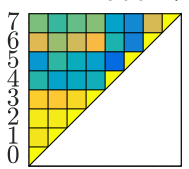

(b) 01234567

Probe 22

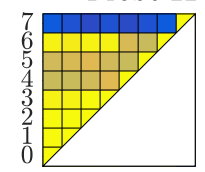

(d) 01234567

Z

FIGURE 10: Correlation between the mixture fraction, $Z$ (denoted by 0 in the axes), and the passive scalars, $Z_{i}^{(a)}$, of the dilution air streams (see Fig. 4(a) for the numbering) for selected locations at the combustor's exit.

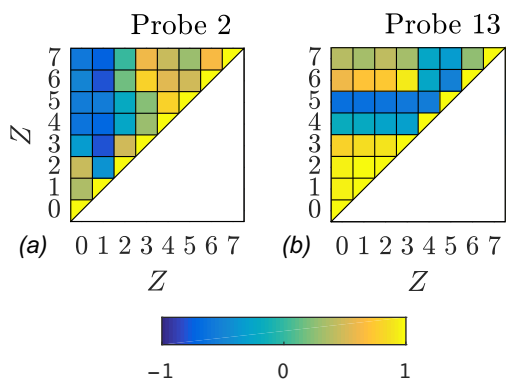

FIGURE 11: Correlation between the mixture fraction, $Z$ (denoted by 0 in the axes), and the passive scalars, $Z_{i}^{(a)}$, of the dilution air streams (see Fig. 4(a) for the numbering) at selected locations in the primary combustion region and downstream of the dilution ports.

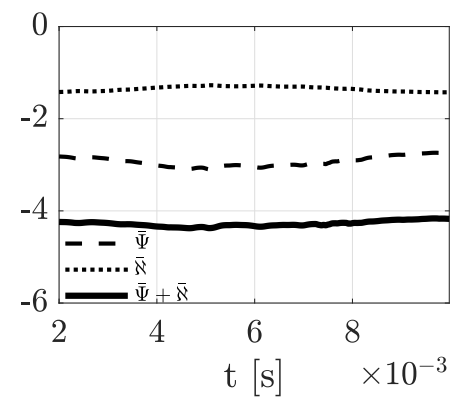

FIGURE 12: Strengths of the acoustic sources due to a compositional inhomogeneity at $\bar{Z}=0.02$ at the combustor's exit, which corresponds to the nozzle inlet. Quantities are cross-sectional averaged. 


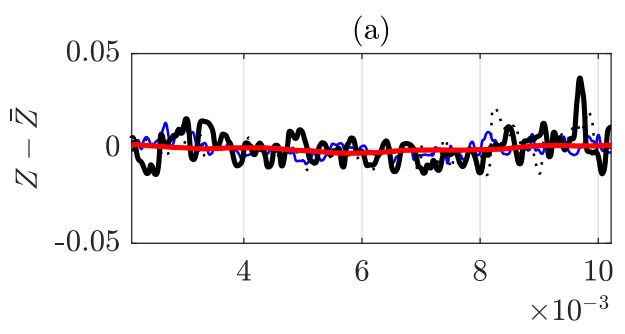

(b)

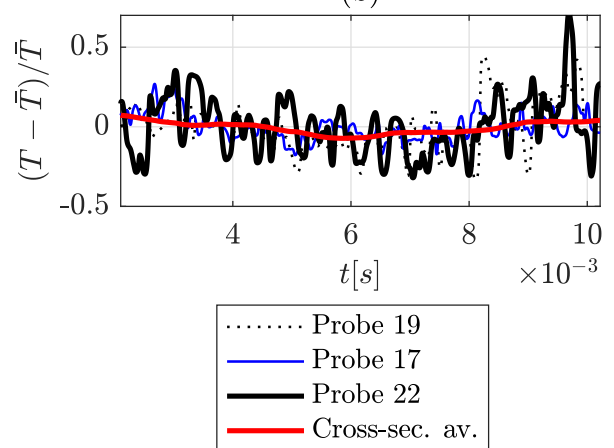

FIGURE 13: Mixture fraction (a) and temperature (b) fluctuations at the combustor's exit. The temperature fluctuation is normalized by the mean temperature, $\bar{T}$. (Fig. 4(a) shows where the probes are placed in the schematic.)

(a)

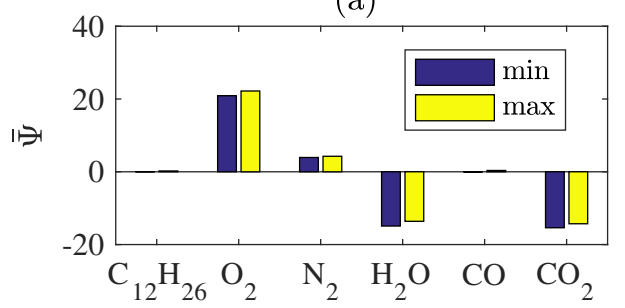

(b)

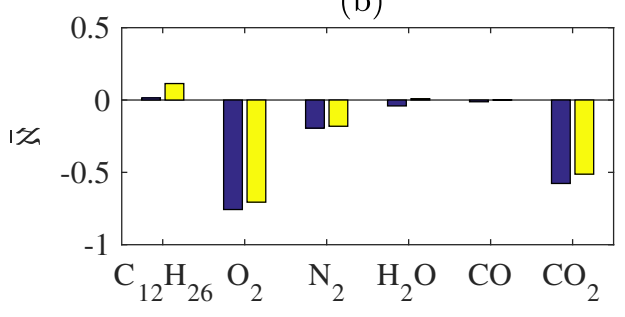

FIGURE 14: Species that most contribute to the strengths of the acoustic sources due to a compositional inhomogeneity. 

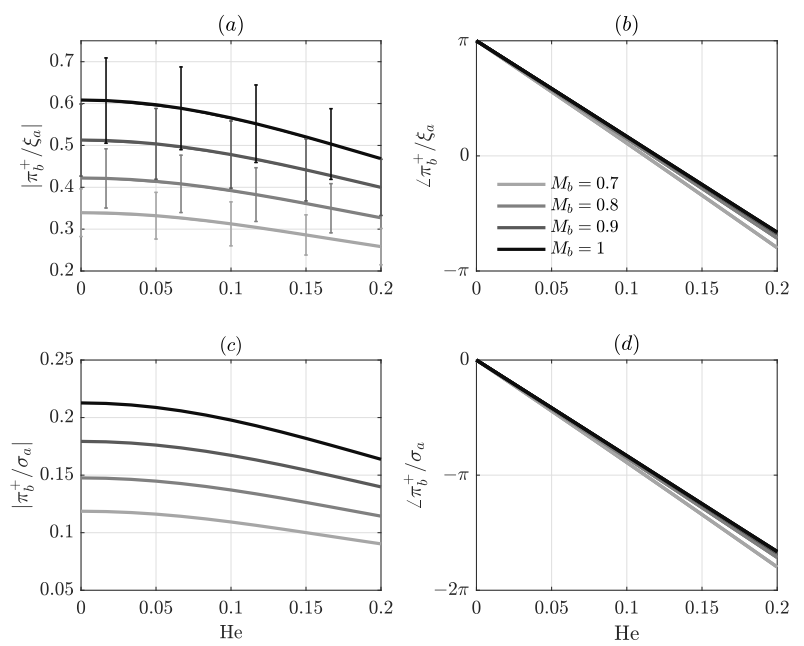

FIGURE 15: Nozzle transfer functions to (a,b) compositional inhomogeneity and (c,d) entropy inhomogeneity coming from the combustor and entering the nozzle. $\pi_{b}^{+}$is the forward-propagating pressure wave at the nozzle's outlet. In (a), the lines correspond to $\bar{\Psi}+\bar{\kappa}=-4.24$, where the min/max values of the bars correspond to $\bar{\Psi}+\bar{\kappa}=-3.92 / \bar{\Psi}+\bar{\kappa}=-4.56$. 